INTERNATIONAL JOURNAL OF APPLIED AND COMPUTATIONAL MATHEMATICS

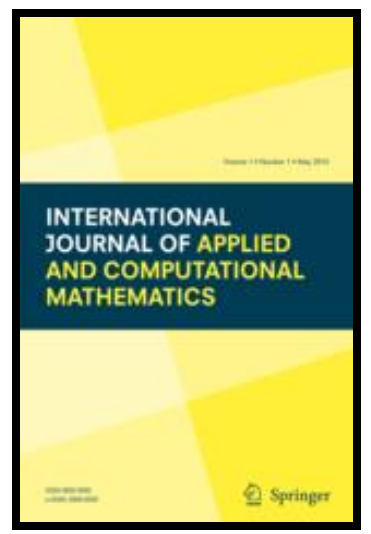

ISSN: 2349-5103 (Print) 2199-5796 (Online)

Publisher: Springer

Accepted July $16^{\text {th }} 2018$

\title{
THEORETICAL STUDY OF OLDROYD-B VISCO-ELASTIC FLUID FLOW THROUGH CURVED PIPES WITH SLIP EFFECTS IN POLYMER FLOW PROCESSING
}

\author{
M. Norouzi ${ }^{1}$, M. Davoodi ${ }^{2}$, O. Anwar Bég ${ }^{3}$ and MD. Shamshuddin ${ }^{*}$ \\ .'Mechanical Engineering Department, Shahrood University of Technology, Shahrood, Iran. \\ 2. School of Engineering, University of Liverpool, Brownlow Hill, Liverpool, L69 3GH, UK. \\ ${ }^{3}$ Aeronautical and Mechanical Engineering, Newton Building, University of Salford, Manchester, M54WT, UK. \\ 4. Department of Mathematics, Vaagdevi College of Engineering, Warangal, Telangana, India.
}

${ }^{*}$ Corresponding author: shammaths@gmail.com

\begin{abstract}
The characteristics of the flow field of both viscous and viscoelastic fluids passing through a curved pipe with a Navier slip boundary condition have been investigated analytically in the present study. The Oldroyd-B constitutive equation is employed to simulate realistic transport of dilute polymeric solutions in curved channels. In order to linearize the momentum and constitutive equations, a perturbation method is used in which the ratio of radius of cross section to the radius of channel curvature is employed as the perturbation parameter. The intensity of secondary and main flows is mainly affected by the hoop stress and it is demonstrated in the present study that both the Weissenberg number (the ratio of elastic force to viscous force) and slip coefficient play major roles in determining the strengths of both flows. It is also shown that as a result of an increment in slip coefficient, the position of maximum velocity markedly migrates away from the pipe center towards the outer side of curvature. Furthermore, results corresponding to Navier slip scenarios exhibit non-uniform distributions in both the main and lateral components of velocity near the wall which can notably vary from the inner side of curvature to the outer side. The present solution is also important in polymeric flow processing systems because of experimental evidence indicating that the no-slip condition can fail for these flows, which is of relevance to chemical engineers.
\end{abstract}

KEYWORDS: Perturbation solution; Oldroyd-B fluid; Slip boundary-condition; Curved pipe; Weissenberg number; Polymer dynamics.

PACS No. : : 46.15.-X, 47.11. +j, 47.10. ab, 44.30. +v, 07.05. Tp.

\section{INTRODUCTION}

The significant diversity of engineering applications of the flow in curved pipes has grown in recent years and these so-called "Dean flows" have emerged in many complex areas of biomedical engineering [1-5] and also chemical engineering [6-7]. Important investigations of the flow in this regime were originally carried out by Dean [8-9] who used a theoretical approach to analyze viscous Newtonian flow in curved tubes. He employed the loose-coiling 
approximation and focused scientific attention on the effects of centrifugal acceleration (arising from the curvature of geometry) in the Navier-Stokes equations. Dean's analytical solutions were based on a perturbation method and highlighted most of the key features of this regime such as Taylor-Görtler secondary flows, corroborating the previous empirical investigation of Eustic [10]. Later studies by Topakoghlu [11] extended their work to the perturbation solutions of both curved circular and annular conduits. Comprehensive reviews of progress in these methods were presented by Berger [12] and later by Guan and Martonen [13] and Naphon and Wongwises [14].

In all of the mentioned works the problem were solved subject to no slip boundary condition. In recent years, slip flow investigations in micro-scale technologies are also developed into an active arena of engineering sciences. Increasingly rich applications of such flows are encountered in biophysical regimes, medical diagnosis, refrigeration systems, chemical reactors, rocket thermal sciences and electronic component cooling mechanisms. The ever-increasing desire for designing the highest performance small scale equipment has led to considerable activity in experimental, analytical and numerical studies of microscale slip flows. These studies principally concentrate on the effects of slip flow in the vicinity of the boundary. This phenomenon generally plays a significant role in micro-scale related surveys of Newtonian fluids. Earlier work by Hooman et al. [15] produced an analytical solution of flow and heat transfer of an isothermal pipe in both hydrodynamically and thermally fully-developed flow via a perturbation method. This analysis focused on the effects of slip flow and heat transfer jump on gaseous flows with variable properties and showed that the presence of a slip condition in micro scale geometries increases the flow rate and consequently heat transfer rates. Contrarily, the temperature jump present around the contact surface of gas and solid decreases the heat transfer value of this scenario. Both phenomena generally show a direct relationship to the dimensionless parameter known as the Knudsen number in gaseous fluids, this parameter is defining as the ratio of mean free molecular distance to an appropriate macro length scale. Slip flows have been examined for a variety of different geometrical configurations including conduits with different cross sections such as rectangular [16], annular [17], hyper-elliptical and regular polygonal cross sections [18]. These studies have also utilized the same slip flow and temperature jump conditions for Newtonian fluids.

In the past three decades, researchers have also directed substantial attention towards the refined modelling industrially complex polymeric liquids with non-Newtonian formulations, particularly viscoelastic fluids. Non-Newtonian transport in curved pipes has been a very active area owing to a tremendous range of applications in polymer processing, biotechnology and food stuffs, slurry transport, petrochemical treatment, pharmacological synthesis, heat exchangers etc. The interesting influences of polymeric fluids related to the first and second normal stresses [19-22] and relaxation and retardation characteristic times [23-27] on the flow behavior and also heat transfer of these fluids has grown into a major field in its own right. An extensive repertoire of analytical, numerical and empirical methods has been successfully implemented in such investigations, yielding important findings and expanding the 
understanding of engineers. The collective influence of characteristics such as elastic force and curvature of geometry and their important role in modifying Taylor-Gortler vortices also make polymeric liquids a suitable potential choice for deploying even in small-scale engineering mechanisms. In the non-slip case, Bowen et al. [28] implemented an approach similar to Topakoglu's [11] to solve the full equations of motion for the creeping flow of upperconvected Maxwell viscoelastic fluids. They also observed that viscoelasticity can generate a pair of secondary flows in the same direction of rotation as that generated by inertial forces. Ebadian [29] and Karahalios and Petrakis [30], using analytical approaches, tried to investigate this issue and they all concluded that the first normal stress can generate a force similar to hoop stress of inertial regime in Newtonian fluids, strengthening the secondary flow. In a following work, Robertson and Muller [24] extended the model in [28] to a more general case by considering viscoelastic inertial flow through circular and annular cross-section curved pipes, reporting in detail the effects of Weissenberg and Reynolds numbers of this regime. In the past, there were several reports in both macro and micro scales that polymeric fluids might experience a slip phenomenon near the interface of the solid and the liquid that this issue can also, potentially, be an important issue in the flow distribution of curved pipes. Hatzikiriakos [31], in a review, discussed different types of slip occurring on molten fluids. In this work, experimental evidences that molten polymers, slip macroscopically at solid surface is reviewed. In following, different types of slip boundary condition is discussed and shown that for some polymers even a second critical wall shear stress exists at which a transition to a stronger slip regime occurs. Kaoullas and Georgiou [32] and Georgiou and Kaoullas [33], showed that in the presence of slip yield stress both the classical no-slip boundary condition and an extension of the Navier's slip phenomenon can be observed depending on the value of the pressure gradient of Newtonian fluids. A similar behavior was observed for viscoelastic Poiseuille flow with slip yield stress [34]. A more detailed study based on numerical and analytical approach for linear and nonlinear Navier slip boundary conditions were carried out in references [35-37].

Due to presence of secondary flow in curved pipe flow of viscoelastic fluids, these geometries are of great importance in designing the highest performance cooling, mixing equipment etc. Motivated by this issue, an important area which requires further analysis is the slip hydrodynamics in proximity to the pipe (conduit) boundary. Therefore, in the present paper, an analytical solution for laminar viscoelastic fluid moving through a curved channel is developed to elucidate the effects of slip on flow characteristics. For this purpose, a perturbation method is employed, in order to derive the closed form velocity field solutions up to second order by considering the curvature ratio as the perturbation parameter. Analytical approaches to viscous flows have been shown to be very powerful, as exemplified in recent work by Siddiqui et al. [38] who employed Bessel and trigonometric functions to solve the biharmonic equation arising in Stokes flow in narrow tubes with wall suction. In the current work, the robust Oldroyd-B model is also implemented as the constitutive rheological equation to simulate polymer liquids with constant viscosity. The effects of the Reynolds 
number, the Weissenberg number, the viscosity ratio and the slip coefficient on viscoelastic flow in a curved channel are studied in detail. The present study provides a useful benchmark for numerical and indeed experimental investigations of Dean Flows of viscoelastic fluids in channels with wall slip and is of relevance to chemical polymer process engineering.

\section{MATHEMATICAL FORMULATION}

\subsection{Dimensionless Group and Constitute Equations}

The physical configuration examined in the present study, namely slip flow of viscoelastic fluids in curved channels is depicted in Fig. 1 , where $r_{0}$ and $R$ are the cross-section radius and the pitch curvature radius, respectively. For the sake of convenience, it is conventional to employ the dimensionless form of the momentum conservation equations. In line with this, the appropriate non-dimensional variables and parameters implemented, are defined below:

$$
\begin{aligned}
& s=\frac{\tilde{s}}{r_{0}}, \quad r=\frac{\tilde{r}}{r_{0}}, \quad u=\frac{\tilde{\underline{v}} \cdot \underline{e}_{r}}{W_{0}}, \quad v=\frac{\underline{\tilde{v}} \cdot \underline{e}_{\phi}}{W_{0}}, w=\frac{\underline{\tilde{v}} \cdot \underline{e}_{s}}{W_{0}}, \quad D=D \frac{r_{0}}{W_{0}}, \\
& \tau^{s}=\frac{r_{0}}{\eta W_{0}} \tilde{\tau}^{s}, \tau^{p}=\frac{r_{0}}{\eta W_{0}} \tilde{\tau}^{p}, P=\frac{r_{0}}{\eta W_{0}} \tilde{P}, W e=\frac{\lambda_{1} W_{0}}{r_{0}}, R e=\frac{\rho W_{0} r_{0}}{\eta}, \delta=\frac{r_{0}}{R}
\end{aligned}
$$

where, $r, \varphi$ and $s$ are the components of toroidal coordinate system. $\underline{e}_{r}, \underline{e}_{\phi}$ and $\underline{e}_{s}$ are the unit vectors in these directions,$r_{0}$ the radius of pipe, $W_{0}$ the maximum velocity of Newtonian fluid in terms of the pressure gradient of $G\left(W_{0}=G r_{0}{ }^{2} / 4 \eta\right), \underline{\tilde{v}}$ the velocity vector, $\tilde{\tau}^{s}$ and $\tilde{\tau}^{p}$ the solvent and polymeric parts of the stress tensor, $\tilde{P}$ the pressure, $\lambda_{1}$ the time constant of fluid, $\eta$ the viscosity, $R e$ the Reynolds number, $\delta$ the curvature ratio, $R$ the curvature radius of the curved pipe, $D$ the rate of deformation tensor and $W_{e}$ the Weissenberg number. As elaborated earlier, the robust, extensively studied, Oldroyd-B constitutive rheological equation is employed to simulate the characteristics of the viscoelastic fluid. This model is also known in the literature as a generalized Jeffreys model (Tripathi et al. [39] and Tripathi and Anwar Bég [40]) and has also been implemented recently in gastric biofluid mechanics and polymer pumping simulations. Essentially this model is derived by substituting the upper-convected derivative with the time derivative of the Jeffreys model (due to its suitability in the simulation of large deformation scenarios). The quasi-linear form of this model renders it more amenable for analyzing the dynamics of dilute polymeric solutions. The Oldroyd-B model is presented herein, based on Newtonian behavior for the solvent and the Upper-Convected Maxwell (UCM) viscoelastic model for polymeric additives such that the $\tilde{\tau}$ stress tensor of this model splits into the sum of $\tilde{\tau}^{s}$ (Newtonian solvent stress) and $\tilde{\tau}^{p}$ (polymeric stress) portions as follows [41]: 


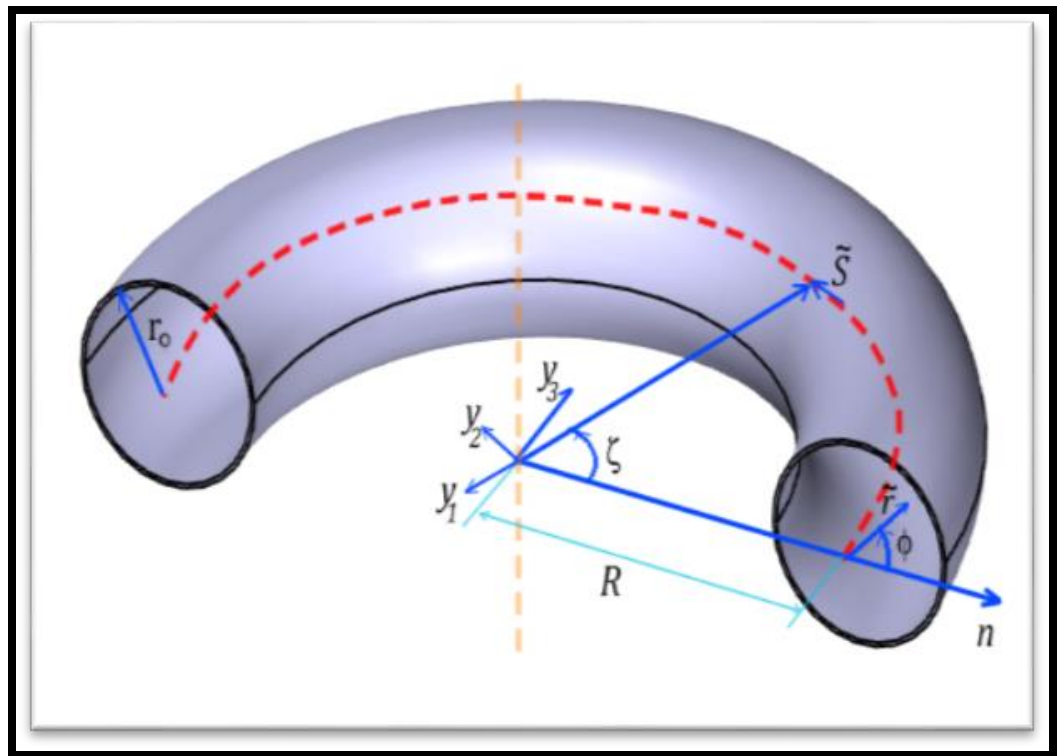

Fig. 1. The geometry of curved channel in current study.

$\tilde{\tau}=\tilde{\tau}^{s}+\tilde{\tau}^{p}$

$\stackrel{\sim}{\tau}^{S}=2 \eta_{S} \tilde{D}, \quad \stackrel{\sim}{\tau} p \stackrel{\nabla}{\tau}+\lambda_{1} \tilde{\tau}^{p}=2 \eta_{p} \tilde{D}$

where $\eta_{s}$ is viscosity of Newtonian solvent and $\eta_{p}$ is viscosity of polymeric portions, respectively. The relationship between employed viscosity of the Oldroyd-B model and its components can be expressed as:

$\eta=\eta_{s}+\eta_{p}$

In general form, the Oldroyd-B constitutive equation represents as [41]:

$$
\tau+\lambda_{1} \stackrel{\nabla}{\tau}=2 \eta\left(\mathrm{D}+\lambda_{2} \stackrel{\nabla}{D}\right)
$$

where $\lambda_{1}$ is relaxation time,is rate of deformation tensor which $\tilde{D}$ ime and is retardation $\mathrm{t} \lambda_{2}$ is the symmetric part of the velocity gradient. The components of the $\tilde{D}$ tensor in the rectangular coordinate system are defined as [41]:

$\tilde{D}_{i j}=\frac{1}{2}\left(\frac{\partial \tilde{v}_{i}}{\partial x_{i}}+\frac{\partial \tilde{v}_{j}}{\partial x_{j}}\right)$

The upper convected derivative of an arbitrary tensor $\tilde{s}_{i j}$ is defined as [41]:

${\stackrel{\nabla}{s_{i j}}}_{i j}=\frac{\partial \tilde{s}_{i j}}{\partial \tilde{t}}+\tilde{v}_{k} \frac{\partial \tilde{s}_{i j}}{\partial x_{k}}-\frac{\partial \tilde{v}_{i}}{\partial x_{k}} \tilde{s}_{j k}-\tilde{s}_{i k} \frac{\partial \tilde{v}_{j}}{\partial x_{k}}$

In the special case where $\lambda_{2}=0$, Eq. (2) is reduced to the upper convected Maxwell (UCM) constitutive equation and when $\lambda_{1}=\lambda_{2}=0$, Oldroyd-B constitutive equation contracts to the classical Newtonian (Navier-Stokes) constitutive equation. 
It is possible to show that if we define the stress of the Odroyd-B model as the summation of UCM additive and Newtonian solvent stresses (Eq. (2)) then the retardation time constant can be derived based on the viscosity ratio and relaxation time constant [41]:

$$
\lambda_{2}=\frac{\eta_{p}}{\eta} \lambda_{1}
$$

\subsection{Governing Equations and Boundary Conditions}

In the presented work, fully developed flow of polymeric solutions using the Oldroyd-B constitutive equation is simulated. The Oldroyd-B model is a suitable choice for viscoelastic materials showing a constant viscosity (Boger Fluids [42]). As it was reported in the literature [31-37], the slip phenomenon can be observed in the interface of polymeric solutions and solid interface. As we know, in the microfluidic devices (Microfluidics deals with the behaviour, precise control and manipulation of fluids that are geometrically constrained to a small, typically sub-millimetre scale [43-45]), the slip situation can more significantly influence in the flow distribution of the material. In accordance with the Fig. 1, owing to the fully developed situation, the derivatives of velocity components and the stress tensor with respect to the angle of curvature $(\zeta)$ are assigned a zero value. The momentum conservation equations in the $r, \phi$ and s directions, for the steady fully-developed flow in a toroidal coordinate system can be represented as [23-24]:

$$
\begin{aligned}
& \frac{\partial(u r B)}{\partial r}+\frac{\partial(v B)}{\partial \phi}=0 \\
& u \frac{\partial u}{\partial r}+\frac{v}{r} \frac{\partial u}{\partial \phi}-\frac{v^{2}}{r}-\frac{\delta}{B} w^{2} \cos \phi=\frac{1}{\operatorname{Re}}\left[-\frac{\partial p}{\partial r}+\frac{\partial \tau_{r r}}{\partial r}+\frac{1}{r} \frac{\partial \tau}{\partial \phi}+\frac{1}{r}\left(\tau_{r r}-\tau_{\phi \phi}\right)\right. \\
& \left.+\frac{\delta}{B}\left(\tau_{r r} \cos \phi-\tau_{r \phi} \sin \phi-\tau_{s S} \cos \phi\right)\right] \\
& u \frac{\partial v}{\partial r}+\frac{v}{r} \frac{\partial v}{\partial \phi}+\frac{v u}{r}+\frac{\delta}{B} w^{2} \sin \phi=\frac{1}{\operatorname{Re}}\left[-\frac{1}{r} \frac{\partial p}{\partial \theta}+\frac{\partial \tau}{\partial r}+\frac{1}{r} \frac{\partial \tau}{\partial \theta}+\frac{2}{r} \tau \phi\right. \\
& \left.+\frac{\delta}{B}\left(\tau_{r \phi} \cos \phi-\tau \phi_{\phi} \sin \phi+\tau_{s S} \sin \phi\right)\right] \\
& u \frac{\partial w}{\partial r}+\frac{v}{r} \frac{\partial w}{\partial \phi}+\frac{\delta}{B} w(u \cos \phi-v \sin \phi)=\frac{1}{\operatorname{Re}}\left[-\frac{\partial p}{\partial z}+\frac{\partial \tau_{s r}}{\partial r}+\frac{1}{r} \frac{\tau}{\partial \phi}+\frac{1}{r} \tau_{s r}\right. \\
& \left.+2 \frac{\delta}{B}\left(\tau_{r s} \cos \phi-\tau_{\phi s} \sin \phi\right)\right]
\end{aligned}
$$

where, $B$ designates the curvature radius of each point and is defined as:

$$
B=1+\delta r \cos (\phi)
$$


The relations between lateral components of velocity and stream function of the secondary flow are defined via Cauchy-Riemann equations, viz:

$u=-\frac{1}{r B} \frac{\partial \psi}{\partial \phi}, \quad v=\frac{1}{B} \frac{\partial \psi}{\partial r}$

Substituting the stream function from of Eq. (11) in Eq. (9a), automatically satisfies the continuity relation. Also, the momentum equation in the pitch direction of curvature can be expressed thus:

$$
\begin{aligned}
\operatorname{Re}\left[\frac{1}{r}\left(\frac{\partial \psi}{\partial r} \frac{\partial w}{\partial \phi}-\frac{\partial \psi}{\partial \phi} \frac{\partial w}{\partial r}\right)-\delta w\left(\frac{1}{r} \frac{\partial \psi}{\partial \phi} \cos \phi+\frac{\partial \psi}{\partial r} \sin \phi\right)\right]= & 4+\frac{\partial \tau_{r s}}{\partial r}+\frac{1}{r}+\frac{\partial \tau_{\phi s}}{\partial \phi}+\frac{1}{r} \tau_{r s} \\
& +2 \delta\left(\tau_{r s} \cos \phi-\tau_{\phi s} \sin \phi\right)
\end{aligned}
$$

In order to achieve possible solutions of velocity components it is necessary to eliminate pressure gradient in the other directions of pipe except in the pitch direction which is already identified. By reducing the $\theta$ derivative of Eq. (9b) and $r$ derivative of equation (9c) from each other, the equation in terms of stream function emerges as follows [23-24]:

$$
\begin{gathered}
\operatorname{Re}\left\{\frac{1}{r}\left(\frac{\partial \psi}{\partial r} \frac{\partial}{\partial \phi} \nabla^{2} \psi-\frac{\partial \psi}{\partial \phi} \frac{\partial}{\partial r} \nabla^{2} \psi\right)+\delta\left[2 w\left(\frac{\partial w}{\partial r} \sin \phi+\frac{1}{r} \frac{\partial w}{\partial \theta} \cos \phi\right)\right.\right. \\
+\left(\sin \phi\left(\frac{3}{r}\left(\frac{\partial \psi}{\partial r}\right)^{2}+\frac{3}{r^{2}} \frac{\partial^{2} \psi}{\partial \phi^{2}} \frac{\partial \psi}{\partial r}-\frac{1}{r^{2}} \frac{\partial \psi}{\partial \phi} \frac{\partial^{2} \psi}{\partial r \partial \phi}+\frac{1}{r^{3}}\left(\frac{\partial \psi}{\partial \phi}\right)^{2}+2 \frac{\partial \psi}{\partial r} \frac{\partial^{2} \psi}{\partial r^{2}}\right)\right. \\
\left.\left.+\cos \phi\left(\frac{3}{r} \frac{\partial^{2} \psi}{\partial r^{2}} \frac{\partial \psi}{\partial \phi}-\frac{1}{r} \frac{\partial \psi}{\partial r} \frac{\partial^{2} \psi}{\partial r \partial \phi}+\frac{3}{r^{2}} \frac{\partial \psi}{\partial r} \frac{\partial \psi}{\partial \phi}+\frac{2}{r^{3}} \frac{\partial \psi}{\partial \phi} \frac{\partial^{2} \psi}{\partial \phi^{2}}\right)\right)\right] \\
\left.+\delta^{2} 3\left[\frac{\sin 2 \phi}{2}\left(\frac{1}{r^{2}}\left(\frac{\partial \psi}{\partial \phi}\right)^{2}-\left(\frac{\partial \psi}{\partial r}\right)^{2}\right)-\cos 2 \phi \frac{1}{r}\left(\frac{\partial \psi}{\partial r} \frac{\partial \psi}{\partial \phi}\right)\right]\right\}= \\
{\left[-\frac{1}{r} \frac{\partial^{2} \tau_{r r}}{\partial r \partial \phi}-\frac{1}{r^{2}} \frac{\partial \tau_{r r}}{\partial \phi}+\frac{1}{r} \frac{\partial^{2} \tau_{\phi \phi}}{\partial r \partial \phi}+\frac{1}{r^{2}} \frac{\partial \tau_{\phi \phi}}{\partial \phi}+\frac{\partial^{2} \tau_{r \phi}}{\partial r^{2}}+\frac{3}{r} \frac{\partial \tau_{r \phi}}{\partial r}-\frac{1}{r^{2}} \frac{\partial^{2} \tau_{r \phi}}{\partial \phi^{2}}\right]} \\
+\delta\left[\begin{array}{l}
\left.\sin \phi\left(\frac{1}{r} \frac{\partial \tau_{r \phi}}{\partial \phi}-\frac{1}{r}\left(\tau_{\phi \phi}-\tau_{r r}\right)+\frac{\partial \tau_{s s}}{\partial r}-\frac{\partial \tau_{\phi \phi}}{\partial r}\right)\right] \\
+\cos \phi\left(\frac{1}{r} \frac{\partial \tau_{s s}}{\partial \phi}-\frac{1}{r} \frac{\partial \tau_{r r}}{\partial \phi}+\frac{2}{r} \tau_{r \phi}-\frac{\partial \tau_{r \phi}}{\partial r}\right)
\end{array}\right]
\end{gathered}
$$

Therefore, the momentum equations are reduced into Equations (12) and (13) where $\nabla^{2}$ denotes the Laplacian operator which is defined as:

$\nabla^{2}=\frac{\partial^{2}}{\partial r^{2}}+\frac{1}{r} \frac{\partial}{\partial r}+\frac{1}{r^{2}} \frac{\partial^{2}}{\partial \phi^{2}}$

It is customary that the slip coefficient would normally be taken to be the same for all directions. In effect, the present approach decomposes the velocity vector into two components, one normal to the surface, with a $v \cdot n=0$ condition, and one tangential, with $v$ - 
subt (the tangential component) $=\beta_{v} \tau_{t}$, where " $t$ " is the vector tangent to the surface. This implies that fluid cannot flow through the wall but that the tangential velocity is proportional to the tangential (shear) component of stress in the direction of flow. Considering the abovementioned description, the boundary condition for slip situation can be written as [46, 31-37]:

$$
\left.w\right|_{r=1}=\beta_{v} \tau_{r s},\left.\quad v\right|_{r=1}=\frac{1}{B} \frac{\partial \psi}{\partial r}=\beta_{v} \tau_{r \varphi},\left.\quad u\right|_{r=1}=-\frac{1}{r B} \frac{\partial \psi}{\partial \varphi}=0
$$

where $\beta_{v}$ denotes the slip coefficient between wall and fluid.

\section{PERTURBATION SOLUTIONS}

Due to the quasi-linear, coupled nature of the momentum conservation equations and the employed constitutive equation, a perturbation method is used to linearize the complex form of these equations. The perturbation parameter in the momentum equations is considered to be the curvature ratio $(\delta=r / R)$. So, in order for this expansion to have a wide range of applicability, it is necessary for any given geometry that the curvature ratio $(\delta)$ be very small. However, according to previous studies this range can be a logical assumption in small scale geometries [47-48]. Here, $\delta=0$ refers to the scenario of a straight pipe. Considering the quasilinear form of the flow distribution in a straight pipe and consequently the absence of secondary flow in this situation $\left(\psi^{(0)}=0\right)$, stream functions start from the first order onwards. The appropriate series forms for the stress tensor, stream function and main velocity are:

$$
w=\sum_{n=0}^{\infty} \delta^{n} w^{(n)}(r, \phi), \quad \psi=\sum_{n=1}^{\infty} \delta^{n} \psi^{(n)}(r, \phi), \quad \tau=\sum_{n=0}^{\infty} \delta^{n} \tau^{(n)}(r, \phi),
$$

\subsection{Flow solution}

Introducing series forms of the parameters defined in Eq. (16) into the momentum equation (12) and arranging coefficients of $\delta^{0}$, the first characteristic equation of the primary (main) velocity is obtained as:

$$
\frac{r^{4} \partial^{2} w^{(0)}}{\partial r^{2}}+4 r^{4}+r^{2} \frac{\partial^{2} w^{(0)}}{\partial^{2} \theta}+r^{3} \frac{\partial w^{(0)}}{\partial r}=0
$$

The zero-order solution of the main velocity $w^{(0)}$ with respect to the slip condition around the wall (15) is:

$$
w^{(0)}=1-r^{2}+2 \beta_{v}
$$

According to the rectilinear flow theorem of Longlois, Rivlin and Pipkin, the solution to the velocity profile of viscoelastic fluids with a constant viscosity (Oldroyd-B fluids in our case) is identical to the Newtonian fluids in rectilinear cases [41]. Although it should be noted that the velocity profile for Oldroyd-B fluids generates a normal stress distribution that consequently 
influence on the pressure distribution which is different than Newtonian cases. So, when the value of $\delta$ is equal to zero, the solution for velocity distribution is the same as the solution of the pipe flow of Newtonian fluids previously reported by Hooman et al. [15] for Newtonian fluid in a straight channel with slip boundary condition. Also, in the case of no-slip, $\beta_{v}=0$, the achieved solution (18) is identical to the no-slip velocity distribution of Newtonian fluid in straight pipes. Arranging terms of order $\delta^{1}$ in the Eq. (13), the first order characteristic equation of the stream function is obtained. The general solution of the characteristic equation of $\psi^{(1)}$ is [23-24]:

$\psi^{(1)}=g_{1}(r) \sin (\phi)$

Applying same method in Eq. (12), the characteristic equation of $w^{(1)}$ is obtained. The closedform of the solution of this equation is derived as a function of the $r$ parameter, multiplying with a cosine function as [23-24]:

$w^{(1)}=f_{1}(r) \cos (\phi)$

Considering the Navier slip boundary condition near the wall (15), $g_{1}(r)$ and $f_{1}(r)$ are calculated which is presented in the appendix with a more detailed information regarding the calculation steps. After substituting the zero and first order velocity components and stress tensors and utilizing the previous approaches for the second order of the main flow and stream function, the characteristic equation of order 2 is obtained. Employing the corresponding slip condition (15), the solutions of the characteristic equations of main flow and stream function equations will be found. We consider the general forms of velocity components as [23-24]:

$\psi^{(2)}=g_{2}(r) \sin (2 \phi)$

$w^{(2)}=f_{20}(r)+f_{22}(r) \cos (2 \phi)$

where $g_{2}(r), f_{20}(r)$ and $f_{22}(r)$ are unknown functions which are laborious and for brevity are not presented, although they are considered in result.

\subsection{Solution of flow rate}


In this section, an analytical solution for the flow rate of Oldroyd-B fluids with slip present near the wall of curved pipe is presented. The dimensionless flow rate through the pipe can be simply presented as:

$$
Q=\int_{0}^{2 \pi} \int_{0}^{1} w r d r d \phi
$$

where $w$ (axial velocity) is considered to be in the form of a perturbation expansion as:

$$
w=w^{(0)}(r)+\delta f_{1}(r) \cos \phi+\delta^{2}\left(f_{20}(r)+f_{22}(r) \cos 2 \phi\right)
$$

Substituting the solutions of velocity components (which are achieved in previous part) into Eqs. (22-23), the equation for the flow rate is obtained which due to the large size of the equation is presented in the appendix.

\section{RESULTS AND DISCUSSION}

In this section, the derived analytical solutions are studied to explore combined effects of slip coefficient, Reynolds and Weissenberg numbers, viscosity ratio and curvature ratio on viscous and viscoelastic flows in curved channels. As it was previously reported in the literature [31-37], the slip situation can occur in both micro and macro scale geometries for polymeric solutions. To estimate a physical range of variable in the current problem, we refer to the aqueous polymer solution used by Xu et. al. [49] with constant viscosity (=285 mPa.s). This solution is prepared by adding $500 \mathrm{ppm}(\mathrm{w} / \mathrm{w})$ of a high-molecular-weight polymer (polyethylene oxide "PEO" of $\mathrm{M}_{\mathrm{w}}=4 \mathrm{e} 06 \mathrm{~g} / \mathrm{mol}$ ) to a more concentrated $42.9 \%(\mathrm{w} / \mathrm{w})$ aqueous solution of the same polymer but of a much lower molecular weight (polyethylene glycol "PEG" $\mathrm{M}_{\mathrm{w}}=8000 \mathrm{~g} / \mathrm{mol}$ ) that provides a polymeric solution with relaxation time of $2.5 \mathrm{~s}$ and density of $1080\left(\mathrm{~kg} / \mathrm{m}^{3}\right)$. Depending on our choice of pressure gradient and the radios of the pipe and the radios of the curvature, we can reach the range of variables which are used in this section. Though, it should be noted, these variables are chosen as an example and one can use the derived formula according to their geometry and rheological request.

The flows of both Newtonian and Oldroyd-B fluids passing through straight pipes display the well-known Poiseuille distribution. In this situation, the velocity contours of main flow are in form of concentric circles (see Fig.2). An interesting phenomenon generated by centrifugal force which causes these circles to deviate from the center line. Some typical sets of main flow contours for Oldroyd-B fluids passing through curved channels are presented in Fig. 2. In this paper, the flow results are presented only in half of the domain due to symmetric distributions about the X-axis. In presented results, the left-hand side of the pipe represents inner side of the curvature and the right-hand side corresponds to the outer side of the curvature. In this figure, the first top four results are related to the classical Newtonian case. The data show that in straight pipes even with the slip effect present, the flow distribution remains concentric. As slip coefficient is increased, maximum value of velocity is elevated and velocity value around 
the wall shows non-zero constant patterns. The remarkable results in the Newtonian cases are related to the scenario with slight curvature ratio of 0.1 . It is evident that the flow distribution around the wall is not constant anymore and can tangibly change along the wall.

The presented data (Figs. 2 and 3) for viscoelastic cases also indicate that an increment in the Reynolds number decreases the dimensionless maximum value of main velocity and causes its position to deviate towards the outer side of the curvature. In contrast, the Weissenberg number, the viscosity ratio and the curvature ratio are observed to enhance the maximum value of main flow and progressively displace its position away from the center toward the outer side of curvature.

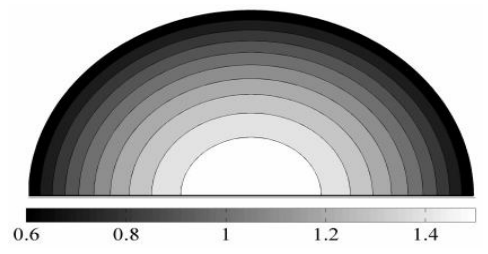

$$
\delta=0 \beta_{v}=0.3(\text { Newtonian })
$$

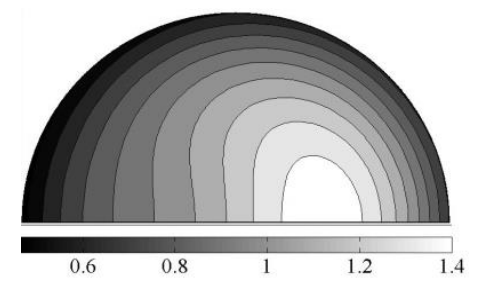

$$
\operatorname{Re}=35 \delta=0.1 \beta_{v}=0.3 \text { (Newtonian) }
$$

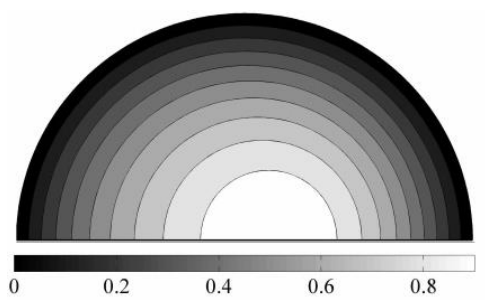

$\beta=.3 \operatorname{Re}=35 \mathrm{We}=3 \delta=0.1 \beta_{v}=0.0$

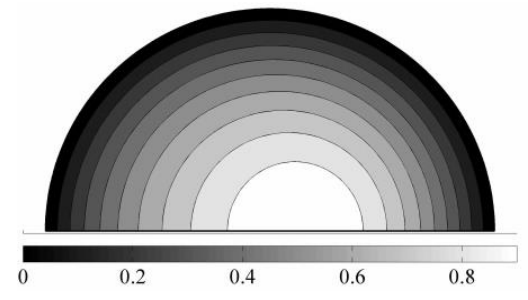

$\beta=.2 \operatorname{Re}=35 \mathrm{We}=5 \delta=0.1 \beta_{v}=0.0$

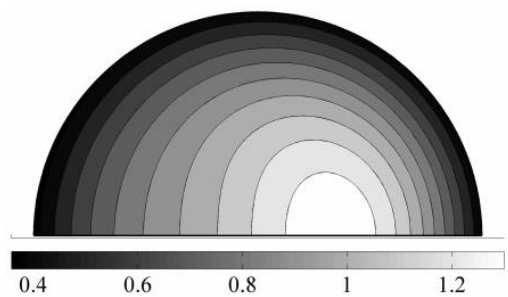

$\beta=.2 \operatorname{Re}=35 \mathrm{We}=3 \delta=0.1 \beta_{v}=0.2$

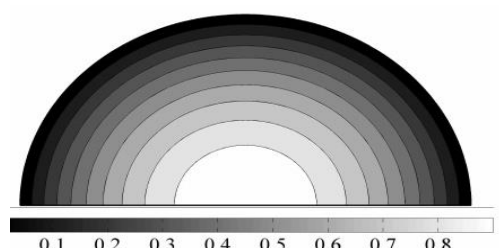

$\delta=0 \beta_{v}=0($ Newtonian $)$

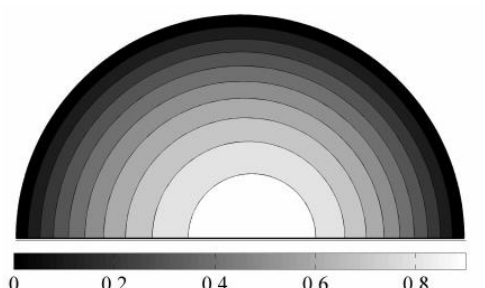

$\operatorname{Re}=35 \delta=0.1 \beta_{v}=0($ Newtonian $)$

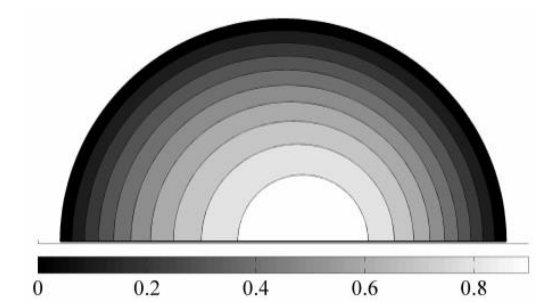

$\beta=.2 R e=35 W e=3 \delta=0.1 \beta_{v}=0.0$

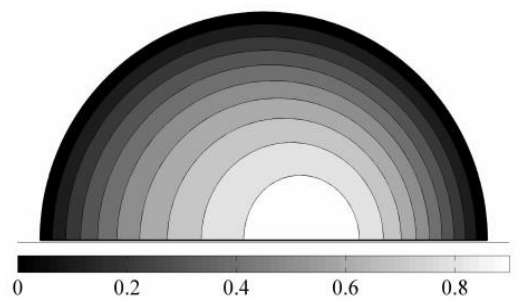


$\beta=.2 \operatorname{Re}=45 \mathrm{We}=3 \delta=0.1 \beta_{v}=0.0$

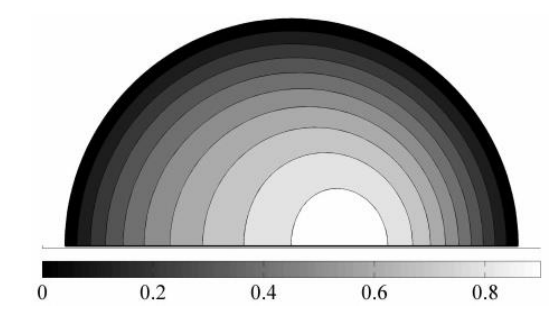

$\beta=.2 \operatorname{Re}=35 \mathrm{We}=3 \delta=0.2 \beta_{v}=0.0$

Fig. 2. Distribution of the velocity $(w)$ in different scenarios.
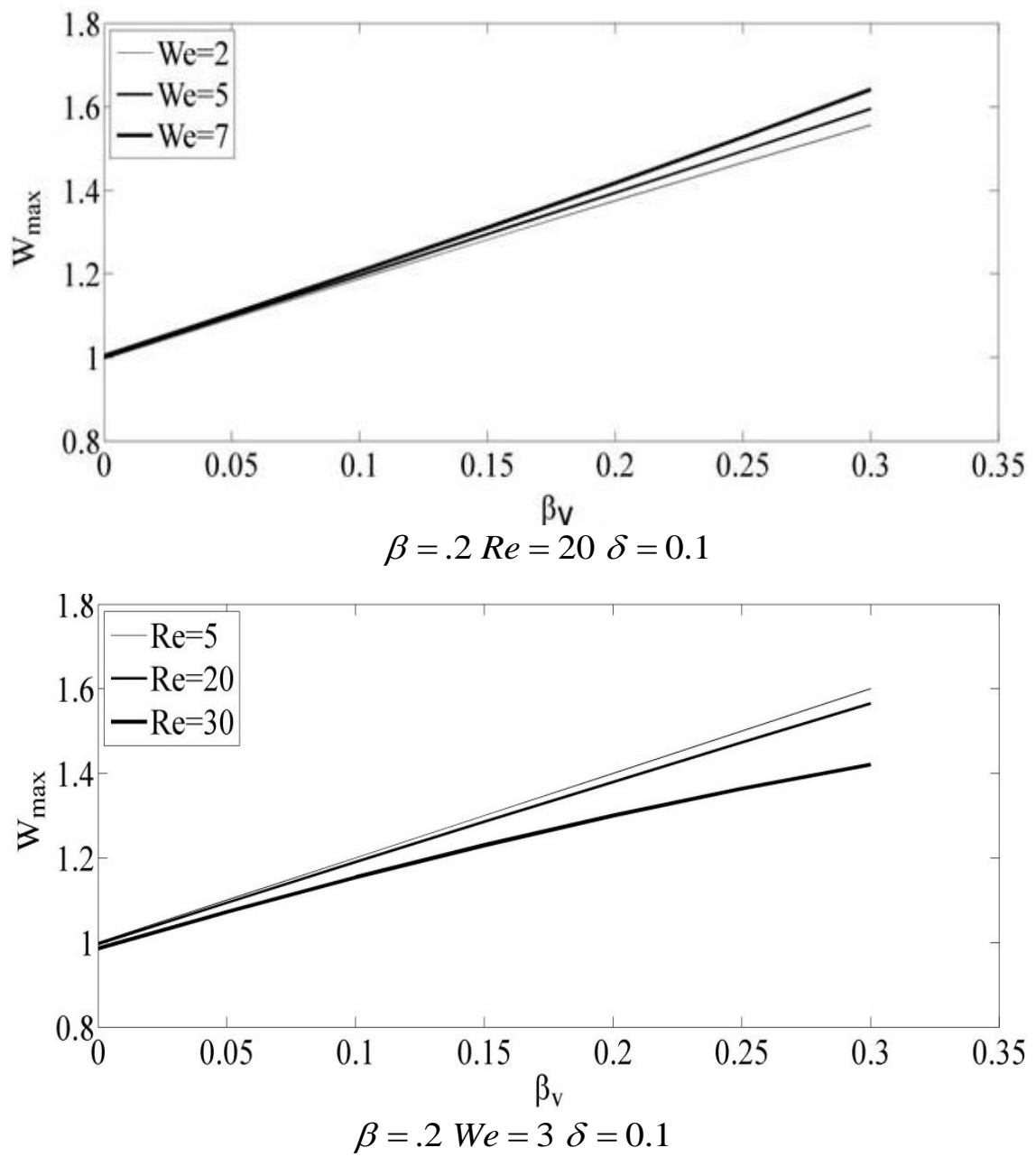


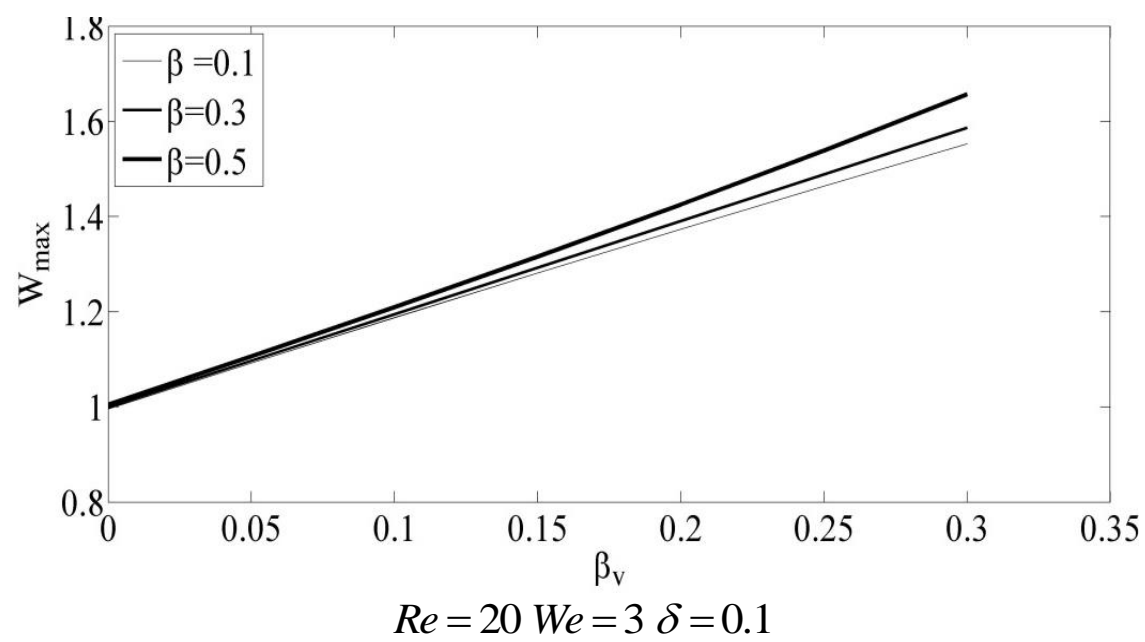

Fig. 3. Maximum value of the main component of the velocity versus the slip coefficient for different conditions.

Previously similar qualitative results were reported by Robertson and Muller [24] for the nonslip situation. Another significant result is associated with the situation in which the flow experiences slip $\left(\beta_{v} \neq 0\right)$. The present perturbation solutions show that in the slip situation, the maximum value of the main flow exceeds that obtained for the non-slip situation. The reason of this phenomenon is related to the choice of the reference velocity which is made in a way that the pressure gradient to be same as the equivalent Newtonian scenario with no slip condition. So, if the pressure drop is fixed, in cases with slip we should expect the flow rate and consequently maximum value of velocity to be increased (as it can also mathematically be observed from equation (18))

Further results for the slip scenario as illustrated in Fig. 4 reveal that an increment in slip.

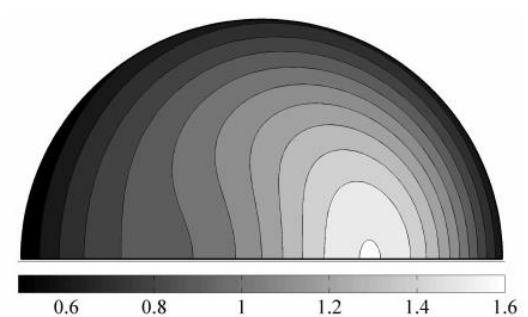

$\beta=.2 \operatorname{Re}=35 \mathrm{We}=3 \delta=0.1 \beta_{v}=0.3$

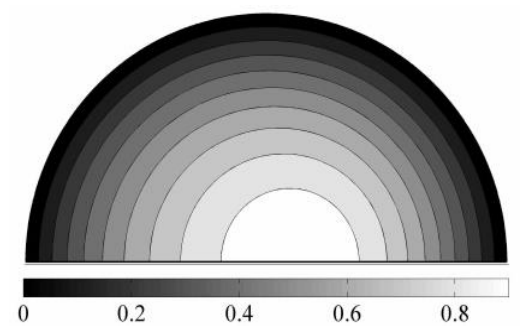

$\beta=.2 \operatorname{Re}=35 W e=3 \delta=0.1 \beta_{v}=0.0$

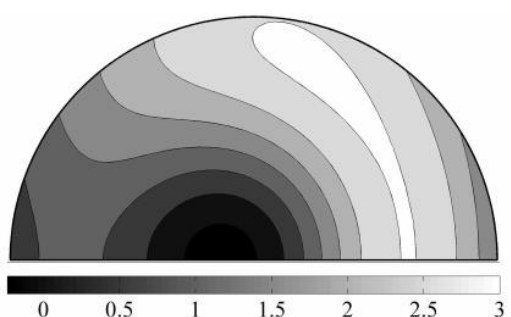

$\beta=.2 \operatorname{Re}=35 \mathrm{We}=3 \delta=0.1 \beta_{v}=1.0$
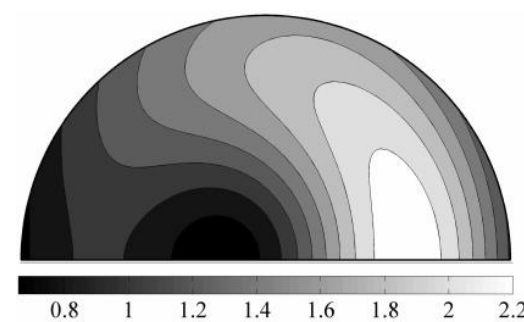

$\beta=.2 R e=35 W e=3 \delta=0.1 \beta_{v}=0.6$

Fig. 4. Effect of slip coefficient on the main component of the velocity field ( $w$ ). 
Coefficient causes the maximum value of velocity to migrate towards the outer side of curvature. In these cases, velocity values vary from the outer side of curvature to the inner side of curvature for non-zero curvature $(\delta \neq 0)$. However, the solutions at higher slip coefficient are qualitatively different. Some similar qualitative patterns of flow for the case of $\beta=0.2, R e=35, W e=3, \delta=0.1$ and $\beta_{v}=0.6$ were previously observed in high Reynolds number flows with no-slip by Robertson and Muller [24]. The data reveals how curiously in high slip situations, the main flow may even show some recirculation phenomena. According to the study of Mashelkar and Devaraja [50] hoop stress $\left(\tau_{s s}\right)$ in Newtonian cases is relatively weak and the pressure gradient in the core region of the pipe is balanced by the centrifugal force. Fan et al. [22], based on an order-of-magnitude analysis for Oldroyd-B Dean flows, derived an equation that estimates the relation between hoop stress $\left(\tau_{s s}\right)$ and centrifugal force. Considering the fact that lateral components of velocity are generally twoorders of magnitude smaller than the main velocity, the momentum equation in the " $n$ " direction of the pipe (see Fig. 1), far from wall and near the core region, can be reduced to:

$\operatorname{Re} \frac{w^{2}}{B} \approx-\frac{\partial p}{\partial n}+\tau_{s s}$

The above equation simply implies that fluid inertia and hoop stress $\left(\tau_{s s}\right)$ are two significant and competitive forces near the core region which contribute to establishing the pressure gradient in " $\mathrm{n}$ " direction for Oldroyd-B fluids in pipes. Fig.5 depicts the hoop stress $\left(\tau_{s s}\right)$ contours for the different scenarios of non-slip and slip in both Newtonian and Oldroyd-B fluids. As observed, the maximum value of the hoop stress in Newtonian case of $R e=35$ and $\delta=0.2$ with non-slip is about 0.03 . In the slip situation $\left(\beta_{v}=0.2\right)$, this value is 3 times greater than that for the non-slip regime. The hoop stress $\left(\tau_{s s}\right)$ value for Oldroyd-B fluid, with properties of $(\beta=0.2$ and $W e=3)$, is found to be around 150 times higher than that computed for the Newtonian cases with slip $\left(\beta_{v}=0.2\right)$. In fact, this difference can even be enhanced 1400 times. Obviously, inertia force, near the wall and its vicinity, is weak (see Fig. 2). Fig. 5 reveals that it is the relatively large axial normal stress near the wall which promotes a secondary flow.

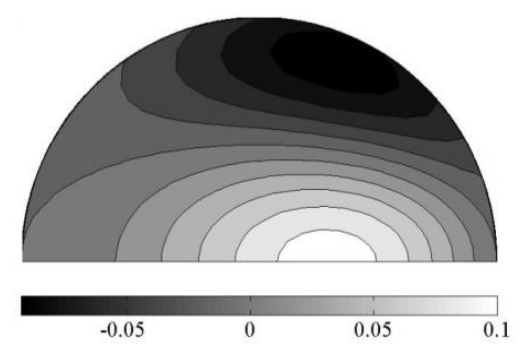

Newtonian Flow $\left(\beta_{v}=0.2\right)$

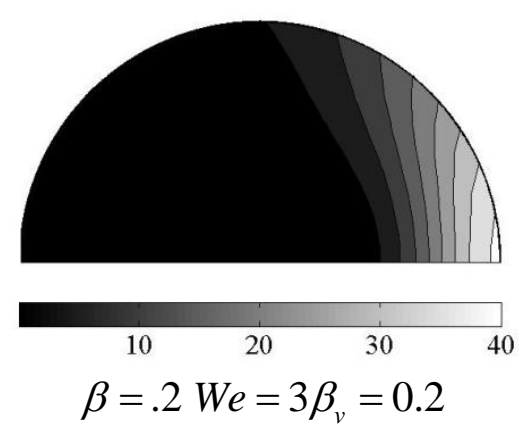

$$
\beta=.2 W e=3 \beta_{v}=0.2
$$




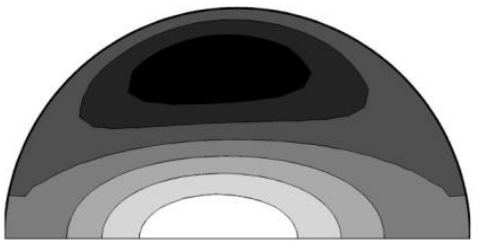

$\begin{array}{llllll}-0.02 & -0.01 & 0 & 0.01 & 0.02 & 0.03\end{array}$

Non-Slip Newtonian Flow $\left(\beta_{v}=0\right)$
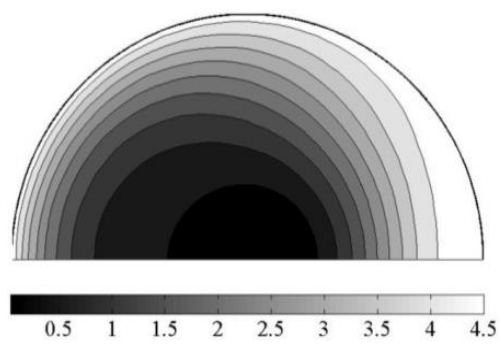

$\beta=.2 W e=3 \beta_{v}=0$

Fig. 5. Contours of hoop stress $\left(\tau_{s s}\right)$ at $\operatorname{Re}=35$ and $\delta=0.2$.

The direction of this secondary flow is observed to be in the same direction as that of the inertial secondary flow. Considering the relationship between hoop stress and intensity of secondary flows, it is estimated that increasing the slip should qualitatively offer a similar effect as the Weissenberg number shows to enhance the intensity of the secondary flow.

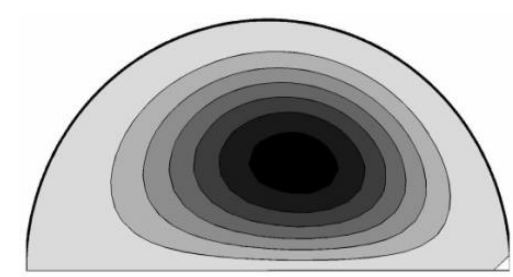

$-0.03-0.025-0.02-0.015-0.01-0.005$

$\beta=.4 R e=35 W e=3 \delta=0.2 \beta_{v}=0.0$
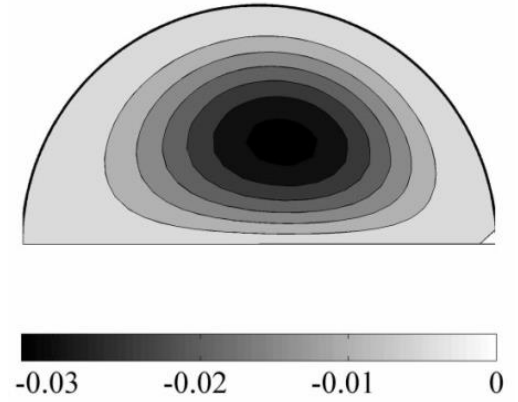

$\beta=.2 \operatorname{Re}=35 \mathrm{We}=5 \delta=0.2 \beta_{v}=0.0$

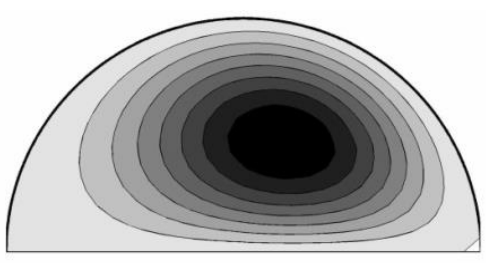

$-0.08-0.07-0.06-0.05-0.04-0.03-0.02-0.01$

$\beta=.2 R e=35 W e=3 \delta=0.2 \beta_{v}=0.2$

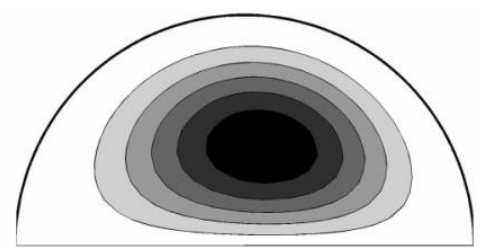

$\begin{array}{lllll}-0.025 & -0.02 & -0.015 & -0.01 & -0.005\end{array}$

$\beta=.2 \operatorname{Re}=35 \mathrm{We}=3 \delta=0.2 \beta_{v}=0.0$ 

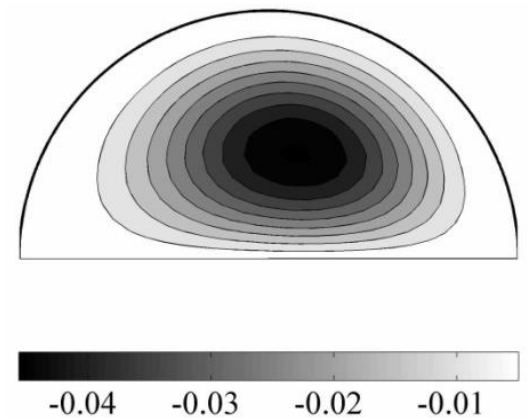

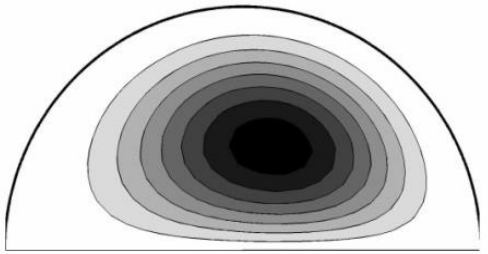

$-0.03$ $-0.01$

$\beta=.2 \operatorname{Re}=45 \mathrm{We}=3 \delta=0.2 \beta_{v}=0.0$

$\beta=.2 \operatorname{Re}=35 \mathrm{We}=3 \delta=0.3 \beta_{v}=0.0$

Fig. 6. Secondary flow contours in different scenarios.

It is well known that flow in straight circular pipe shows rectilinear distribution without any secondary flow in both non-Newtonian and Newtonian fluids. Fig. 6 depicts the secondary flow shape of different fluids passing through a curved channel. These results confirm that an increment in Weissenberg and Reynolds numbers and curvature and viscosity ratios intensifies the secondary flows, a trend which has also previously been documented by Robertson and Muller [24]. The present study has also shown that some significant and novel results are related to the slip regimes. Fig. 7 shows that an increment in slip coefficient intensifies secondary flows.

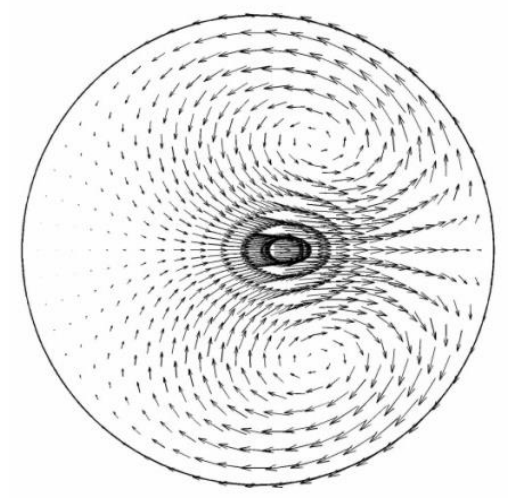

$\psi_{\text {max }}=0.1114$

$\beta=.2 \operatorname{Re}=35 \mathrm{We}=3 \delta=0.2 \beta_{v}=0.3$

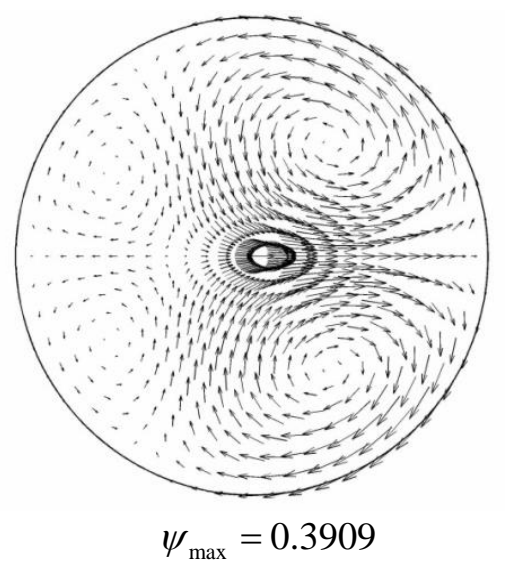

$\beta=.2 R e=35 W e=3 \delta=0.2 \beta_{v}=1.0$ 

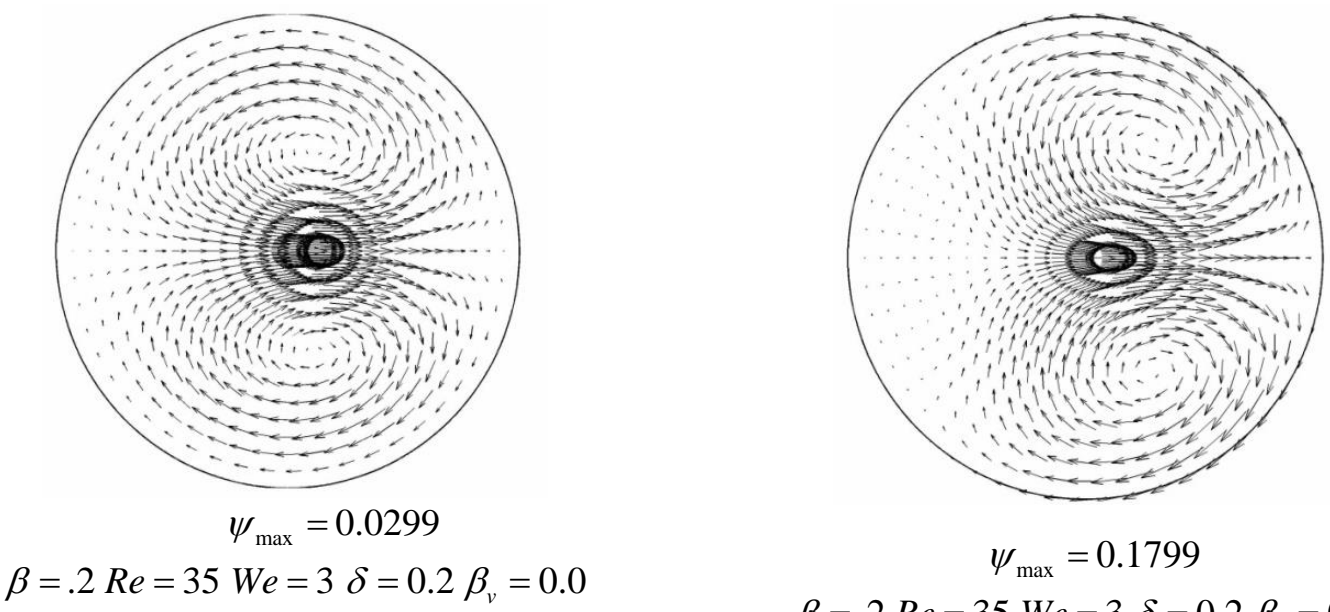

$$
\beta=.2 \operatorname{Re}=35 \mathrm{We}=3 \delta=0.2 \beta_{v}=0.5
$$

Fig. 7. Effect of slip coefficient on secondary flow and inducing second pair of vortices.

The data clearly demonstrate that the velocity distribution around the wall is not zero (constant) any longer and markedly varies from the inside toward the outside of wall. As previously presented in Fig. 4, the scenario with high slip situation can lead to the initiation of a recirculation phenomenon.

Here, the solutions show that lateral components of velocity also change their own direction. It appears that another pair of secondary flow in the opposite direction to the previous one is produced. Fig. 8 reveals that an increment in other parameters can strengthen both secondary flows arising. Fig. 9 shows the flow rate of Oldroyd-B viscoelastic fluids in curved pipes and indicates that an increment in slip coefficient, viscosity ratio and Weissenberg number enhances the flow rate whereas increasing the Reynolds number results in the converse effect and decreases flow rate.
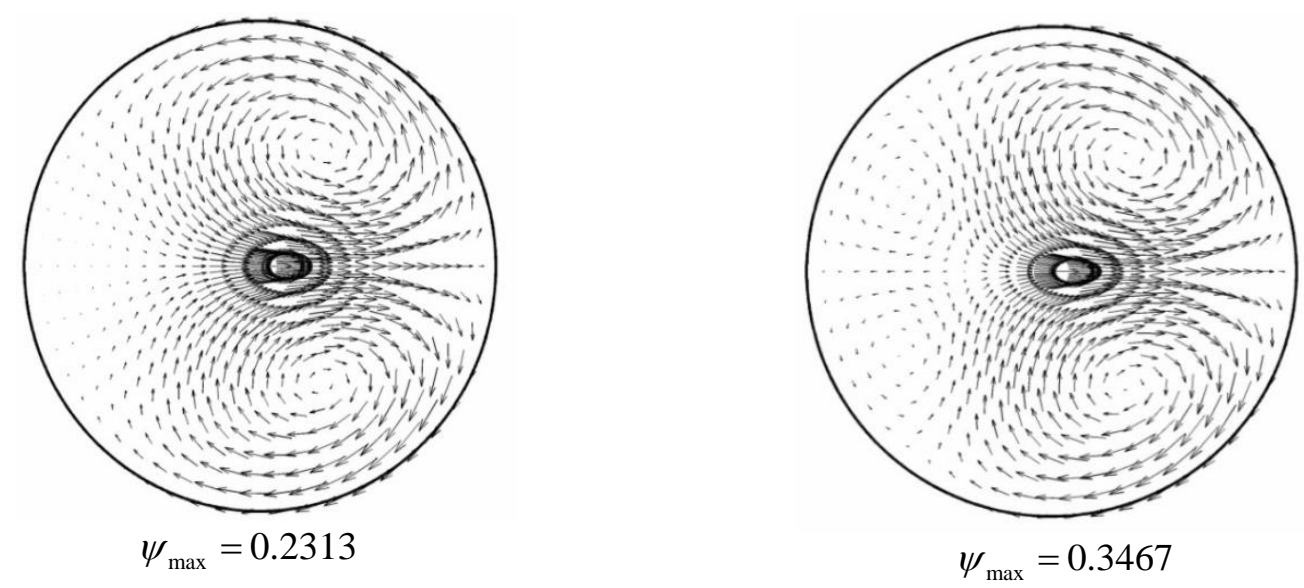

$\beta=.2 \operatorname{Re}=35 \mathrm{We}=5 \delta=0.2 \beta_{v}=0.6$

$\beta=.2 R e=45 W e=5 \delta=0.2 \beta_{v}=0.6$ 

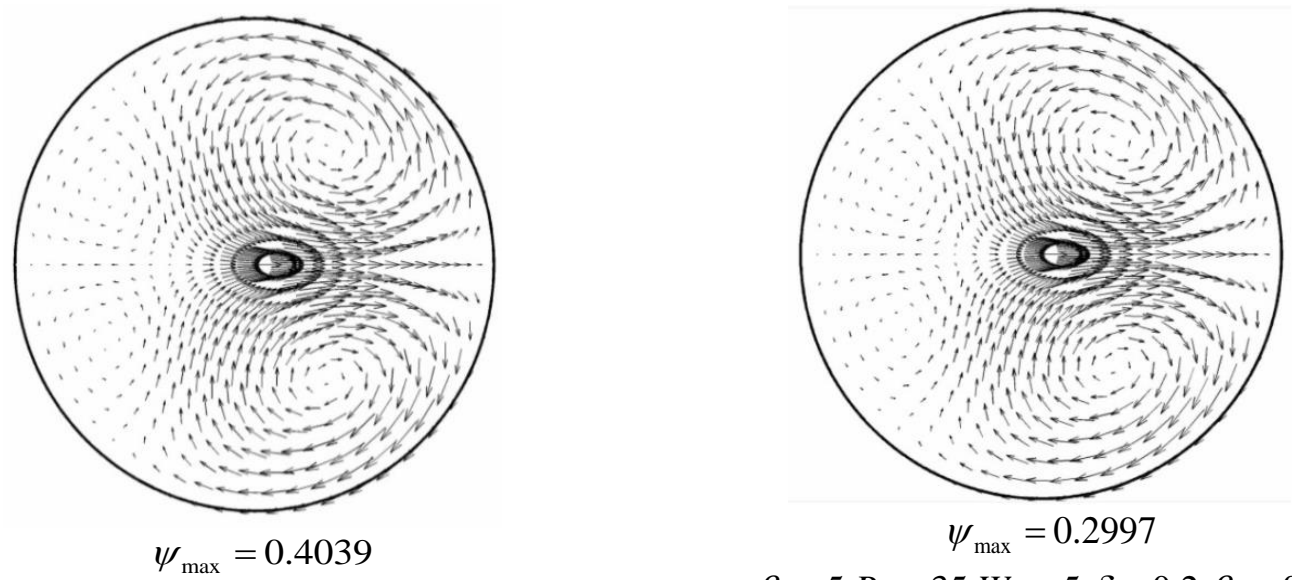

$\beta=.2 R e=35 W e=5 \delta=0.3 \beta_{v}=0.6$
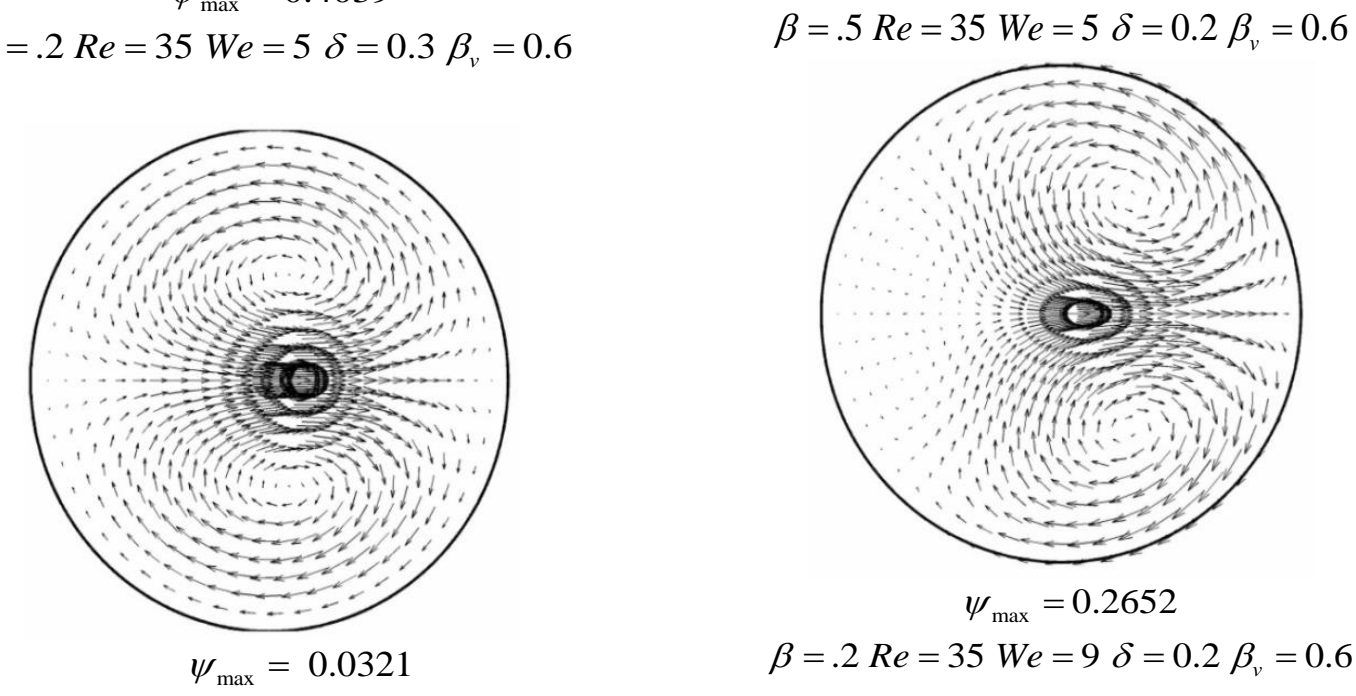

$\beta=.2 R e=35 W e=5 \delta=0.2 \beta_{v}=0$

Fig. 8. Mutual effects of different parameters on inducing second pair of vortices.

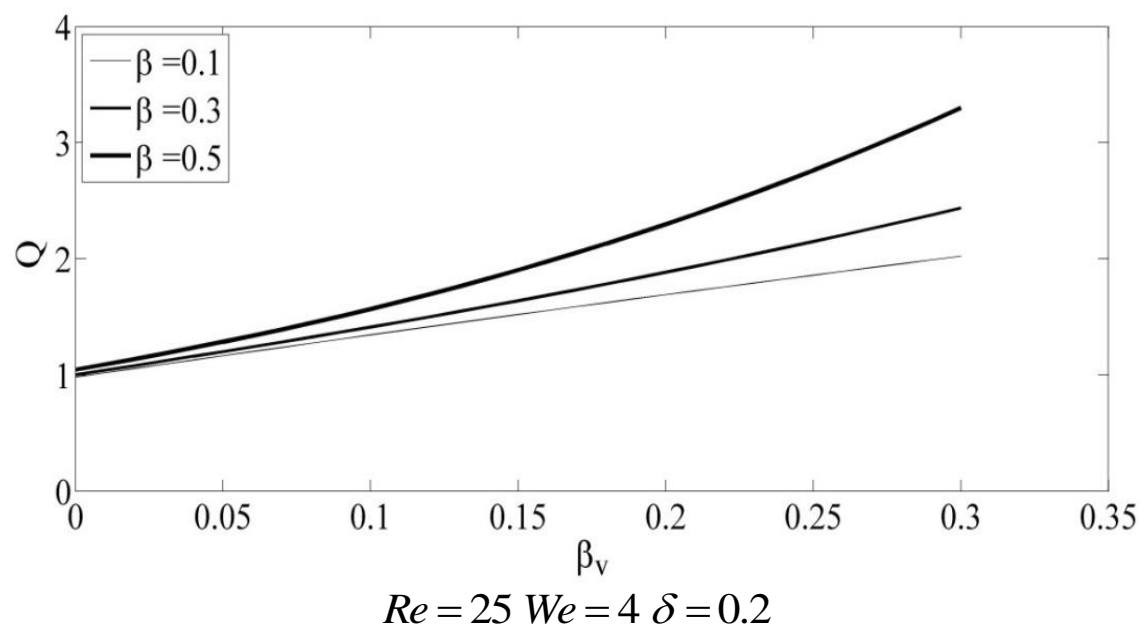



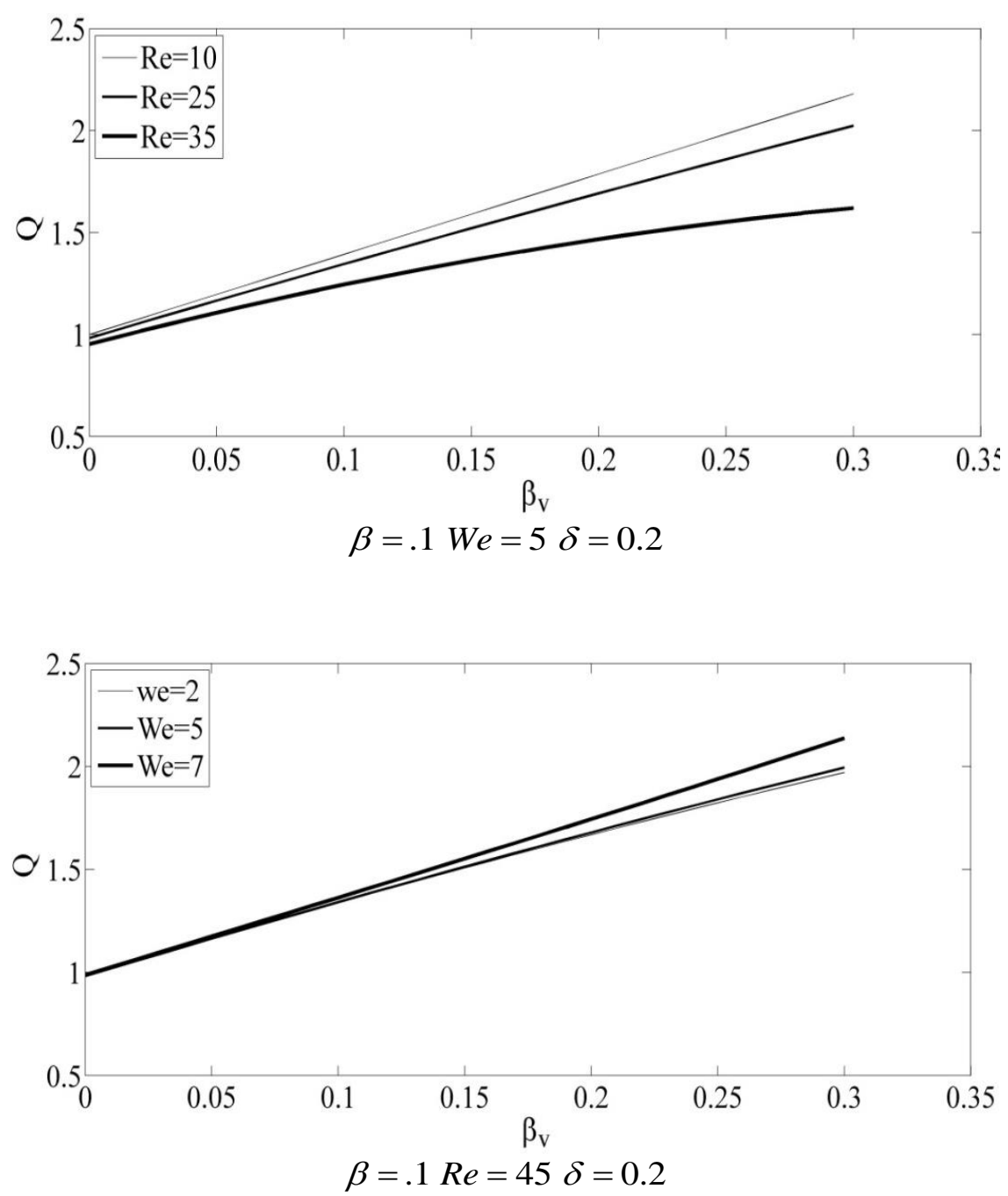

Fig. 9. Dimensionless flow rate variation versus slip coefficient.

\section{CONCLUSIONS}

Analytical solutions have been derived using a perturbation technique, for the viscoelastic fluid passing through a curved channel of circular cross-section, with a Navier slip boundary condition present. A solution to the flow with slip boundary condition up to second order of the expansion has been obtained to estimate characteristics of the flow regime. The Oldroyd-B rheological model is employed due to suitability with liquids possessing constant stretch histories. The earlier analysis of Fan et al. [22], proved that the flow characteristics of Oldroyd-B fluids in curved pipes has a direct relationship with the hoop stress $\left(\tau_{s s}\right)$ component of the stress tensor. The present analytical results reveal that slip coefficient greatly effects the intensity and distribution of this stress component, similar to Weissenberg number. A slight enhancement in the slip coefficient between the wall and fluid can highly intensify the hoop stress. In the slip situation, a non-uniform distribution of velocity around the wall is observed which can significantly vary from the outside of 
curvature toward inside of the wall. Further analysis shows that an increment in $R e, W e, \beta$ and $\delta$ results in strengthening the secondary flows and causes the location of maximum velocity to migrate towards the outside of curvature. The present solutions provide a useful benchmark for numerical simulations and also experimental investigations related to Oldroyd-B viscoelastic flows in curved channels. Future work will consider alternate viscoelastic models including the Williamson [51] and Jeffery [52] models which also provide good approximations to polymer flows.

\section{Acknowledgements}

We express our gratitude to Professor Morton Denn of the Levich Institute, University of Delaware, USA, for his invaluable help and guidance regarding the present research. Furthermore, the authors acknowledge the comments of both reviewers which have served to improve the present article.

\section{Compliance with Ethical Standards:}

The ethical standards are considered in writing this paper. The authors have no conflict of interest with anybody or any organizations/companies.

\section{References}

[1] W H Finlay, Phys. Fluids A 1854 (1989).

[2] T Hayat, S Noreen and A Alsaedi, J. Mech. Med. Biol. 121 (2012).

[3] M M Hoque, M M Alam, M Ferdows and O. Anwar Bég, Proc. Inst. Mech. Eng. H: J Eng. in Medicine 2271155 (2013).

[4] M Z Keshavarz and L Kadeem, Med. Eng. Phys. 33315 (2011).

[5] N Ali, M Sajid, Z Abbas and T Javed, Eur. J. Mech.-B: Fluids 29387 (2010).

[6] V Kumar, M Agarwal and K D P Nigam, Chem. Eng. Sci. 615742 (2006).

[7] K C Cheng and M Akiyama, Appl. Sci. Res. 29401 (1974).

[8] W R Dean, Phil. Mag. 4208 (1927).

[9] W R Dean, Phil. Mag. 5673 (1928).

[10] J Eustice, Proc. Roy. Soc-London series A 85119 (1911).

[11] H C Topakoglu, USSR J. Math. Mech. 161321 (1967).

[12] S A Berger, L Talbot and L S Yao, Ann. Rev. Fluid Mech. 15461 (1983).

[13] X Guan and T B Martonen, Aerosol. Sci. Tech. 26485 (1997).

[14] P Naphon and S Wongwises, Renew. Sus. Energy Rev. 10463 (2006).

[15] K Hooman, F Hooman and M Famouri, Int. Commun. Heat Mass Transfer 36192 (2009).

[16] W A Khan and M M Yovanovich, AIAA J. Thermophysics Heat Transfer 22352 (2008).

[17] Z Duan, Y S Muzychka, ASME J Heat Transfer 1301 (2008).

[18] A Tamayol and M Bahrami, ASME J Fluids Eng. 1321 (2010).

[19] M Norouzi, M Davoodi, O Anwar Bég and A A Joneidi, Int. J. Therm. Sci. 6961 (2013).

[20] M Norouzi, M H Kayhan, C Shu and M R H Nobari, J. Non-Newtonian Fluid Mech. 165323 (2010).

[21] M Norouzi, M Davoodi and O Anwar Bég, Int. J. Therm. Sci. 9090 (2015).

[22] Y Fan, R I Tanner, N Phan-Thien, J. Fluid Mech. 440327 (2001).

[23] W Jitchote and A M Robertson, J. Non-Newtonian Fluid Mech. 9091 (2000).

[24] A M Robertson and S J Muller, Int. J. Non-linear Mech 313 (1996).

[25] C F Hsu and S V Patankar, AIChemE J. 28610 (2004).

[26] N Phan-Thien and R Zheng, ZAMP: J. Appl. Math. Phys. 41766 (1990).

[27] M Zhang, X Shen, J Ma, B Zhang, Int. J. Heat and Mass Transfer 51661 (2008).

[28] P J Bowen, A R Davies and K Walters, J. Non-Newtonian Fluid Mech 38113 (1991). 
[29] M A Ebadian, ASME J. Appl. Mech. 571073 (1990).

[30] G T Karahalios and M A Petrakis, Acta Mechanica 881 (1991).

[31] S G Hatzikiriakos, Progress in Polymer Sci. 37(4) 624 (2012).

[32] G Kaoullas and G C Georgiou, J. Non-Newtonian Fluid Mech. 19724 (2013).

[33] G C Georgiou and G Kaoullas, Meccanica 48(10) 2577 (2013).

[34] Y Damianou, M Philippou, G Kaoullas and G C Georgiou, J. Non-Newtonian Fluid Mech. 20324 (2014).

[35] L L Ferrás, A M Afonso, M A Alves, J M Nóbrega and F T Pinho, J. Non-Newtonian Fluid Mech. 21280 (2014).

[36] L L Ferrás, J M Nóbrega and F T Pinho, J. Non-Newtonian Fluid Mech. 17576 (2012).

[37] Y M Joshi and M M Denn, J. Non-Newtonian Fluid Mech. 114185 (2003).

[38] A.M. Siddiqui et al. Alexandria Engineering Journal, 56.1, 105 (2017).

[39] D Tripathi, O Anwar Bég and J Curiel-Sosa, Computer Methods In Biomech. Biomed. Eng. 17433 (2014).

[40] D Tripathi and O Anwar Bég, Int. J. Thermal Sci. 7041 (2013).

[41] R B Bird and R C Armstrong, O Hassager and C F Curtiss, Dynamics of Polymeric liquids: Volume 1- Fluid Mechanics, New York: Wiley (1977).

[42] D F James, Annual Review of Fluid Mech. 41129 (2009).

[43] W M Abed, D W Richard, J C D David and J P Robert, Int. J. Heat and Mass Transfer 88790 (2015).

[44] K Zografos, F Pimenta, M A Alves and M S N Oliveira, Biomicrofluidics 10(4) 043508 (2016).

[45] G K Satish, G Srinivas, D Li, C Stephane and R K Michael, Heat Transfer and Fluid Flow in Minichannels and Microchannels, Elsevier (2005).

[46] C L M H Navier, Mem. Acad. Sci. Inst. France 6389 (1827).

[47] W M Abed, D W Richard, J C D David and J P Robert, J. Non-Newtonian Fluid Mech. 23168 (2016).

[48] W M Abed, D W Richard, J C D David and J P Robert, Int. J. Heat and Mass Transfer 88790 (2015).

[49] H Xu, A Clarke, J P Rothstien and R J Poole, Appl. Phys. Lett. 108241602 (2016).

[50] R A Mashelkar and G V Devarajan, Trans. Inst. Chem. Engrs. 54100 (1976).

[51] M.M. Bhatti, M.M. Rashidi, Journal of Molecular Liquids, 221, 567-573 (2016).

[52] M.M. Bhatti, A. Zeeshan, R. Ellahi, Microvascular Research, 110, 32-42 (2017). 


\section{APPENDIX}

Due to the quasi-linear, coupled nature of the momentum conservation equations, a perturbation method is used. The perturbation parameter in the momentum equations is considered to be the curvature ratio $\left(\delta=\frac{r}{R}\right)$. Considering the rectilinear form of the flow distribution in a straight pipe and consequently the absence of secondary flow in this situation $\left(\psi^{(0)}=0\right)$, stream functions start from the first order onwards. The appropriate series forms for the stress tensor, stream function and main velocity are:

$w=\sum_{n=0}^{\infty} \delta^{n} w^{(n)}(r, \phi), \quad \psi=\sum_{n=1}^{\infty} \delta^{n} \psi^{(n)}(r, \phi), \quad \tau=\sum_{n=0}^{\infty} \delta^{n} \tau^{(n)}(r, \phi)$

Introducing (A-1) into the momentum equation and arranging coefficients of $\delta^{0}$, the first characteristic equation of the primary (main) velocity is obtained as:

$$
\frac{r^{4} \partial^{2} w^{(0)}}{\partial r^{2}}+4 r^{4}+r^{2} \frac{\partial^{2} w^{(0)}}{\partial^{2} \theta}+r^{3} \frac{\partial w^{(0)}}{\partial r}=0
$$

The zero-order solution of the main velocity $w^{(0)}$ with respect to the slip condition around the wall is:

$$
w^{(0)}=1-r^{2}+2 \beta_{v}
$$

Upon substituting Eq. (A-3) into the Oldroyd-B Constitutive Equation the zeroth order solution os the stress components can be calculated as:

$$
\tau_{r s}{ }^{(0)}=\frac{\partial w^{(0)}}{\partial r}, \tau_{\phi s}{ }^{(0)}=\frac{1}{r} \frac{\partial w^{(0)}}{\partial \phi}, \tau_{s s}{ }^{(0)}=2 W e \beta\left(\left(\frac{\partial w^{(0)}}{\partial r}\right)^{2}+\left(\frac{1}{r} \frac{\partial w^{(0)}}{\partial \phi}\right)^{2}\right)
$$

After collecting the coefficient of $\delta$ in Eqs. 17 and 16, the following equation can be obtained, respectively:

$$
\begin{aligned}
\left(2 \operatorname{Re} w^{(0)} \frac{\partial w^{(0)}}{\partial r}-\frac{\partial \tau_{s s}{ }^{(0)}}{\partial r}\right) \sin \phi= & -\frac{1}{r} \frac{\partial^{2} \tau_{r r}{ }^{(1)}}{\partial r \partial \phi}-\frac{1}{r^{2}} \frac{\partial^{2} \tau_{r r}{ }^{(1)}}{\partial \phi}+\frac{1}{r} \frac{\partial^{2} \tau_{\phi \phi}{ }^{(1)}}{\partial r \partial \phi}+\frac{1}{r^{2}} \frac{\partial \tau_{\phi \phi}{ }^{(1)}}{\partial \phi} \\
& +\frac{\partial^{2} \tau_{r \phi}{ }^{(1)}}{\partial r^{2}}+\frac{3}{r} \frac{\partial \tau_{r \phi}{ }^{(1)}}{\partial r}-\frac{1}{r^{2}} \frac{\partial^{2} \tau_{r \phi}{ }^{(1)}}{\partial \phi^{2}} \\
\nabla^{2} w^{(1)}=4 \cos \phi-\operatorname{Re}\left(\frac{\partial w^{(0)}}{\partial r} \frac{\partial \psi^{(1)}}{\partial \phi}\right) & -W e \beta \frac{1}{r}\left(\frac{\partial^{3} w^{(0)}}{\partial r^{3}} \frac{\partial \psi^{(1)}}{\partial \phi}-\frac{\partial^{2} w^{(0)}}{\partial r^{2}} \frac{\partial^{2} \psi^{(1)}}{\partial r \partial \phi}+3 \frac{1}{r} \frac{\partial w^{(0)}}{\partial r^{2}} \frac{\partial \psi^{(1)}}{\partial \phi}\right. \\
- & \frac{\partial w^{(0)}}{\partial r} \frac{\partial^{3} \psi^{(1)}}{\partial \phi \partial r^{2}}+\frac{1}{r} \frac{\partial w^{(0)}}{\partial r} \frac{\partial^{2} \psi^{(1)}}{\partial \phi \partial r}-3 \frac{1}{r^{2}} \frac{\partial w^{(0)}}{\partial r} \frac{\partial \psi^{(1)}}{\partial \phi}-\frac{\partial w^{(0)}}{\partial r} \frac{\partial^{3} \psi^{(1)}}{\partial \phi^{3}}
\end{aligned}
$$

Using the perturbation series presented in eqn. $(A-1)$ in constitutive equation, expressions for first order of stress tensor components can be obtained as:

$$
\begin{aligned}
& \tau_{r r}{ }^{(1)}=-2 \frac{\partial}{\partial r}\left(\frac{1}{r} \frac{\partial \psi^{(1)}}{\partial \phi}\right), \tau_{\phi \phi}{ }^{(1)}=2 \frac{\partial}{\partial r}\left(\frac{1}{r} \frac{\partial \psi^{(1)}}{\partial \phi}\right), \\
& \tau_{r \phi}{ }^{(1)}=\frac{\partial^{2} \psi^{(1)}}{\partial r^{2}}-\frac{1}{r} \frac{\partial \psi^{(1)}}{\partial \phi}-\frac{1}{r^{2}} \frac{\partial^{2} \psi^{(1)}}{\partial \phi^{2}}
\end{aligned}
$$

Solution to the equation (A-5nad A-6), considering Eq. (A-7) will be in the form of $\psi^{(1)}=g_{1}(r) \sin \phi$ and $w^{(1)}=f_{1}(r) \cos \phi$ which the function $g_{1}(r)$ and $f_{1}(r)$ are calculated using the slip boundary condition. 


$$
\begin{aligned}
& g_{1}(r)=-(1 / 48) r^{5} \operatorname{Re}-(1 / 12) r^{5} W e \beta-(1 / 24) r^{5} \beta_{v} \operatorname{Re}+(1 / 288) \operatorname{Re} r^{7}-(1 / 72) r \operatorname{Re} /\left(1+2 \beta_{v}\right) \\
& -(1 / 9) r \beta_{v} \operatorname{Re} /\left(1+2 \beta_{v}\right)+(2 / 3) r^{3} \beta_{v} W e \beta /\left(1+2 \beta_{v}\right)+(1 / 3) r^{3} \beta_{v}^{2} \operatorname{Re} /\left(1+2 \beta_{v}\right) \\
& -(1 / 12) r W e \beta /\left(1+2 \beta_{v}\right)-(1 / 2) r \beta_{v} W e . \beta /\left(1+2 \beta_{v}\right)-(1 / 4) r \beta_{v}{ }^{2} \operatorname{Re} /\left(1+2 \beta_{v}\right) \\
& +(1 / 32) r^{3} \operatorname{Re} /\left(1+2 \beta_{v}\right)+(1 / 6) r^{3} W e \beta /\left(1+2 \beta_{v}\right)+(3 / 16) r^{3} \beta_{v} \operatorname{Re} /\left(1+2 \beta_{v}\right) \\
& f_{1}(r)=\left(1920 r^{5} W e^{2} \beta^{2} /\left(23040 \beta_{v}+11520\right)-40 r^{7} \operatorname{Re}^{2} \beta_{v} /\left(23040 \beta_{v}+11520\right)+\right. \\
& 320 r^{5} \operatorname{Re}^{2} \beta_{v}^{2} /\left(23040 \beta_{v}+11520\right)+(11 / 32) r \beta W e \operatorname{Re} \beta_{v} /\left(2 \beta_{v}^{2}+3 \beta_{v}+1\right)+ \\
& 640 r^{5} \beta W e \operatorname{Re} /\left(23040 \beta_{v}+11520\right)+3840 r^{5} \beta_{v} W e^{2} \beta^{2} /\left(23040 \beta_{v}+11520\right)+ \\
& 2560 r^{5} \beta W e \operatorname{Re} \beta_{v} /\left(23040 \beta_{v}+11520\right)-5760 r^{3} \beta W e \operatorname{Re} \beta_{v} /\left(23040 \beta_{v}+11520\right) \\
& -120 r^{7} \beta W e \operatorname{Re} /\left(23040 \beta_{v}+11520\right)-(5 / 3) r W e^{2} \beta_{v}^{2} \beta^{2} /\left(2 \beta_{v}{ }^{2}+3 \beta_{v}+1\right)- \\
& 960 r^{3} \beta W e \operatorname{Re} /\left(23040 \beta_{v}+11520\right)+30 r^{5} \operatorname{Re}^{2} /\left(23040 \beta_{v}+11520\right)- \\
& (5 / 6) r W e \beta \beta_{v}{ }^{3} \operatorname{Re} /\left(2 \beta_{v}{ }^{2}+3 \beta_{v}+1\right)-10 r^{7} \operatorname{Re}^{2} /\left(23040 \beta_{v}+11520\right)- \\
& (3 / 4) r /\left(2 \beta_{v}^{2}+3 \beta_{v}+1\right)+4 r \beta_{v}^{3} /\left(2 \beta_{v}^{2}+3 \beta_{v}+1\right)-(5 / 2) r \beta_{v}^{2} / \\
& \left(2 \beta_{v}{ }^{2}+3 \beta_{v}+1\right)-(15 / 4) r \beta_{v} /\left(2 \beta_{v}{ }^{2}+3 \beta_{v}+1\right)+(19 / 11520) r \operatorname{Re}{ }^{2} / \\
& \left(2 \beta_{v}{ }^{2}+3 \beta_{v}+1\right)+8640 r^{3} /\left(23040 \beta_{v}+11520\right)+r^{9} \operatorname{Re}^{2} /\left(23040 \beta_{v}+11520\right) \\
& -40 r^{3} \operatorname{Re}^{2} /\left(23040 \beta_{v}+11520\right)+17280 r^{3} \beta_{v} /\left(23040 \beta_{v}+11520\right)-320 r^{3} \operatorname{Re}^{2} \beta_{v}{ }^{\prime} \\
& \left(23040 \beta_{v}+11520\right)+(1 / 6) r W e^{2} \beta^{2} /\left(2 \beta_{v}{ }^{2}+3 \beta_{v}+1\right)+(127 / 1920) r \operatorname{Re}^{2} \beta_{v}{ }^{2} / \\
& \left(2 \beta_{v}{ }^{2}+3 \beta_{v}+1\right)+(209 / 11520) r \operatorname{Re}^{2} \beta_{v} /\left(2 \beta_{v}{ }^{2}+3 \beta_{v}+1\right)+(7 / 96) r \operatorname{Re}^{2} \beta_{v}{ }^{3 /} \\
& \left(2 \beta_{v}{ }^{2}+3 \beta_{v}+1\right)-7680 r^{3} \beta W e \operatorname{Re} \beta_{v}^{2} /\left(23040 \beta_{v}+11520\right)-3840 r^{3} W e^{2} E^{2} / \\
& \left(23040 \beta_{v}+11520\right)+2 r^{9} \operatorname{Re}^{2} \beta_{v} /\left(23040 \beta_{v}+11520\right)+180 r^{5} \operatorname{Re}^{2} \beta_{v} /\left(23040 \beta_{v}+11520\right) \\
& -15360 r^{3} \beta_{v} W e^{2} \beta^{2} /\left(23040 \beta_{v}+11520\right)-40 r^{7} \operatorname{Re}^{2} \beta_{v}{ }^{2} /\left(23040 \beta_{v}+11520\right)+ \\
& (11 / 288) r \beta W e \operatorname{Re} /\left(2 \beta_{v}{ }^{2}+3 \beta_{v}+1\right)+(77 / 144) r \beta W e \operatorname{Re} \beta_{v}{ }^{2} /\left(2 \beta_{v}{ }^{2}+3 \beta_{v}+1\right)- \\
& 720 r^{3} \operatorname{Re}^{2} \beta_{v}{ }^{2} /\left(23040 \beta_{v}+11520\right)+(7 / 6) r \beta_{v} W e^{2} \beta^{2} /\left(2 \beta_{v}{ }^{2}+3 \beta_{v}+1\right) \\
& +1920 r^{5} \beta W e \operatorname{Re} \beta_{v}^{2} /\left(23040 \beta_{v}+11520\right)-240 r^{7} \beta W e \operatorname{Re} \beta_{v} /\left(23040 \beta_{v}+11520\right)
\end{aligned}
$$

The same method can be used to drive the characteristic equation of order 2 which due to large size of equations are not presented here but are included once results are reported. The solution to the characteristic equation of order two are in the forms of [23-24]:

$$
\begin{aligned}
& \psi^{(2)}=g_{2}(r) \sin (2 \phi) \\
& w^{(2)}=f_{20}(r)+f_{22}(r) \cos (2 \phi)
\end{aligned}
$$

\section{Solution of Flow Rate}

The dimensionless flow rate through the pipe can be simply presented as: 
$Q=\int_{0}^{2 \pi} \int_{0}^{1} w r d r d \phi$

where $\mathrm{w}$ (axial velocity) is considered to be in the form of a perturbation expansion as:

$w=w^{(0)}(r)+\delta f_{1}(r) \cos \phi+\delta^{2}\left(f_{20}(r)+f_{22}(r) \cos 2 \phi\right)$

(A-13)

Substituting the solutions of velocity components into Eqs. (A12-A13), the following equation for the flow rate is readily arrived at:

$\tilde{Q}_{s}=\frac{\pi r_{o}^{2}}{8 \eta} G$

(A-14)

where, $Q_{s}$ is the dimensionless flow rate in a straight stationary pipe with the same pressure gradient and this value is equal to $\pi / 2$. The magnitude of this parameter is:

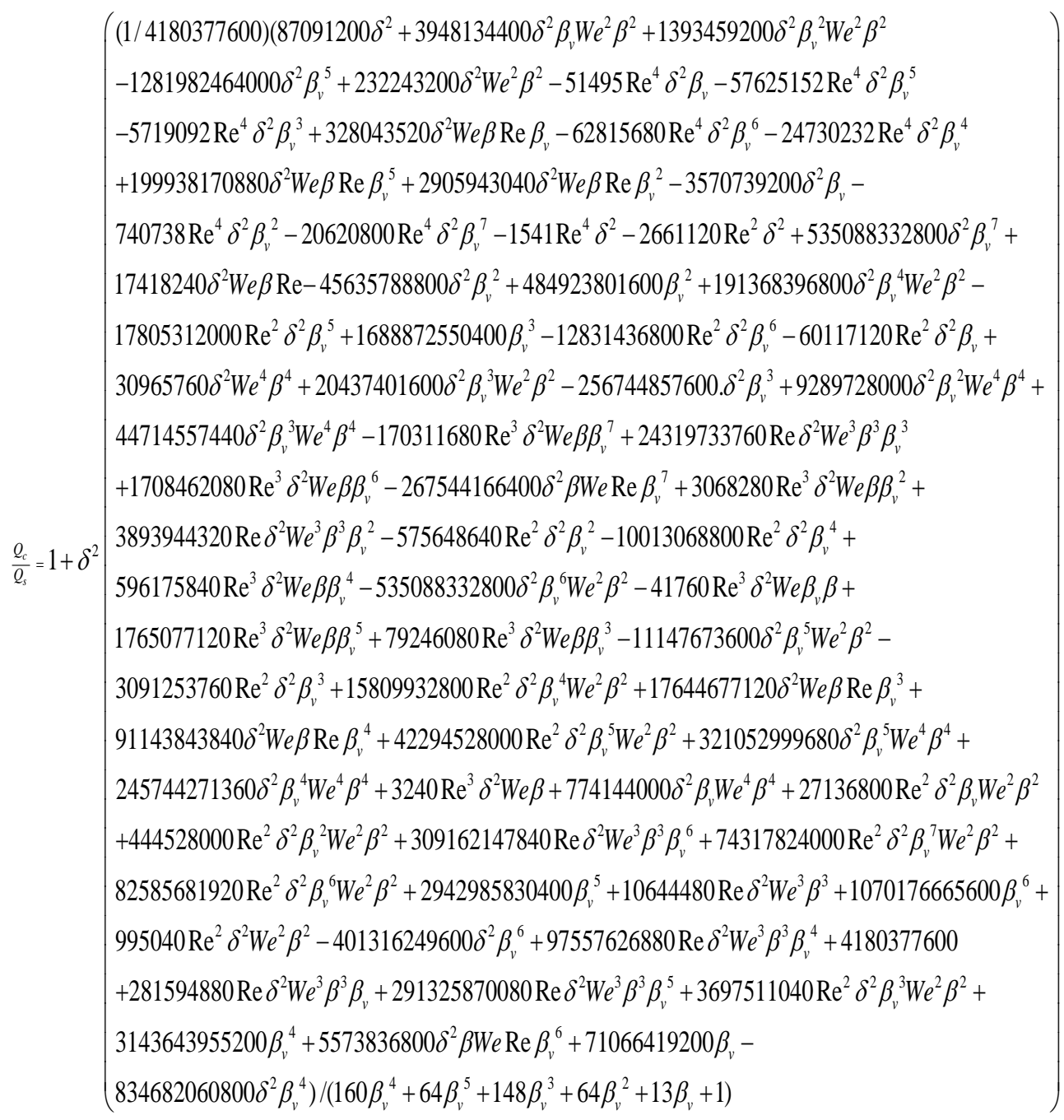

